\title{
Quantitative sampling of atomic-scale electromagnetic waveforms
}

\author{
D. Peller ${ }^{1, \#}$, C. Roelcke ${ }^{1}$, L. Z. Kastner ${ }^{1}$, T. Buchner ${ }^{1}$, A. Neef ${ }^{1}$, J. Hayes ${ }^{1}$,
} F. Bonafée, ${ }^{2, \#}$ D. Sidler ${ }^{2}$, M. Ruggenthaler ${ }^{2}$, A. Rubio ${ }^{2,3, *}$, R. Huber ${ }^{1, *} \&$ J. Repp ${ }^{1}$

${ }^{1}$ Department of Physics, University of Regensburg, 93040 Regensburg, Germany

${ }^{2}$ Max Planck Institute for the Structure and Dynamics of Matter, Center for Free Electron Laser Science, 22761 Hamburg, Germany

${ }^{3}$ Center for Computational Quantum Physics, Simons Foundation Flatiron Institute, New York, NY 10010 USA and Universidad del País Vasco, UPV/EHU- 20018 San Sebastián, Spain

Tailored nanostructures can confine electromagnetic waveforms in extremely sub-wavelength volumes, opening new avenues in lightwave sensing and control down to sub-molecular resolution. Atomic light-matter interaction depends critically on the absolute strength and the precise time evolution of the near field, which may be strongly influenced by quantum mechanical effects. Measuring atom-scale field transients, however, has been out of reach. Here, we introduce quantitative atomic-scale waveform sampling in lightwave scanning tunnelling microscopy to resolve a tip-confined near-field transient. Our parameter-free calibration employs a singlemolecule switch as an atomic-scale voltage standard. While salient features of the far-to-near-field transfer follow classical electrodynamics, we develop a comprehensive understanding of the atomicscale waveforms with time-dependent density-functional theory. The simulations validate our calibration and confirm that single-electron tunnelling ensures minimal back-action of the measurement process on the electromagnetic fields. Our observations access an uncharted domain of nano-opto-electronics where local quantum dynamics determine femtosecond atomic near fields. 
22 The ability to map out the temporal shape of optical carrier waves, like an oscilloscope for light ${ }^{1-4}$, 23 has prompted a vibrant field of research exploring light-matter interaction faster than a cycle of light ${ }^{5-11}$.

24 Electro-optic sampling and streaking techniques visualize lightwaves by stroboscopically scanning them 25 with ultrashort gate pulses. When this idea is combined with scanning near-field optical microscopy ${ }^{12,13}$, 26 radiation scattered from the tip-confined near field can be detected electro-optically to monitor lightwaves 27 with combined $10 \mathrm{~nm}$ spatial and subcycle temporal resolution. Meanwhile, sophisticated nanostructures 28 including nanoantennas ${ }^{14-17}$, nanoparticles ${ }^{18}$, nanogaps ${ }^{19-21}$, and $\operatorname{tips}^{22-24}$ have facilitated near-field 29 confinement, to volumes even smaller than the skin depth in metals ${ }^{16,20,25}$. Such tailored near fields have 30 advanced (bio)chemical sensing ${ }^{26}$, light harvesting ${ }^{26}$, lightwave electronics ${ }^{22-25,27-31}$, nanoimaging ${ }^{12,13,27-31}$ 31 and spectroscopy ${ }^{12,13,32,33}$ down to sub-molecular resolution ${ }^{28,34}$. Importantly, atomic light-matter 32 interaction depends critically on the absolute field strength and the precise temporal evolution of the 33 oscillating carrier near field ${ }^{12,15,16,22-25,27-32,35-39}$, both of which may be strongly influenced by quantum 34 mechanical effects, such as tunnelling ${ }^{36,39}$. Yet, measuring electromagnetic transients on atomic length 35 scales, where novel non-classical dynamics have been predicted to shape local fields ${ }^{36,39}$, is highly 36 challenging.

37 Lightwave-induced electron emission from sharp tips ${ }^{22-24}$ has been used to reveal fascinating 38 interferences of electron trajectories. The distribution of electron kinetic energies encodes complex 39 dynamics including quiver motion and interferometric backscattering. While this approach has resolved 40 tip-confined transients in a broad range of settings, the interaction between field-emitted electrons and a 41 test waveform is not strictly localized in space, hence it is challenging to fully reconstruct the local near 42 field. In lightwave-driven scanning tunnelling microscopy (STM) $)^{27-31,38}$, conversely, terahertz (THz) 43 pulses control local electron tunnelling with sub-cycle temporal resolution. Optical gating of 44 semiconductor samples has been used to extract near-field waveforms under the assumption that the 45 carriers relax extremely rapidly and that the steady-state conductance applies equally for femtosecond 46 bursts of a tunnelling current ${ }^{38}$. Yet, detection of near-field waveforms with atomic resolution has 47 remained an open challenge, let alone the measurement of calibrated absolute fields. State-selective 
tunnelling in ultrafast single-molecule STM indeed combines sub- $\AA$ localization with spectroscopic

49 orbital sensitivity ${ }^{28}$, suggesting individual molecules as a quantitative atomic near-field sensor.

\section{Quantitative waveform sampling with an atom-scale voltage sensor}

51 Here, we introduce waveform sampling in ultrafast single-molecule STM as the first method to

52 locally detect tip-confined near-field waveforms at combined sub-cycle temporal and atom-scale spatial

53 scales. The detection scheme is quantitative without free parameters and builds on lightwave STM, where

54 the oscillating carrier wave of ultrashort $\mathrm{THz}$ pulses serves as an ultrafast biasing voltage controlling

55 single-electron tunnelling. To this end, the THz far field is focused onto the junction of an STM,

56 consisting of a metallic probing tip (tungsten) located a few angstroms ( $\AA$ ) over a conductive substrate

57 (Fig. 1a). This arrangement acts as a THz antenna (Fig. 1b), for which the confinement into the $\AA$-scale

58 tunnelling gap enhances the field by several orders of magnitude. While the coupling efficiency,

59 plasmonic propagation and near-field screening as well as geometrical phase retardation could be

60 described by classical electrodynamics, the tip-confined fields will also depend on atomic details of the

61 geometry ${ }^{21}$ and quantum effects like tunnelling. This currently renders a priori predictions of the near-

62 field waveform inside the STM junction impossible.

63 To map out the actual waveform locally and quantitatively, we introduce an atomically small voltage

64 gauge into the tunnelling gap: a single-molecule switch. It can be activated only if the voltage $V(t)$ that

65 results from the local field $E(t)$ in the tunnelling gap exceeds a critical threshold. Ultrafast temporal

66 resolution is obtained by superimposing the near-field waveform $V_{\mathrm{NF}}(t)$, which is to be determined, with a

$67 \mathrm{THz}$ gate pulse $V_{\text {gate }}(t)$. To this end, we generate pairs of identical THz waveforms with an adjustable

68 delay time $\tau$ in a Michelson interferometer and strongly decrease the amplitude of one pulse in a pair

69 using crossed polarizers. In Fig. $1 \mathrm{c}, V_{\mathrm{NF}}(t)$ is set five times weaker than the corresponding gate waveform

$70 V_{\text {gate }}(t-\tau)$ (Fig. 1d). When the pulses interfere (Fig. 1e), the waveform of the test transient offsets the field

71 crest of the gate. Because $V_{\text {gate }}(t)$ is much stronger than $V_{\mathrm{NF}}(t)$, and thanks to the very nonlinear threshold

72 behaviour of the switch, $V_{\text {gate }}(t)$ can be used as a subcycle probe for $V_{\mathrm{NF}}(t)$. In fact, the peak of the sum 
73 voltage $V_{\text {peak }}(\tau)$ traces out the instantaneous waveform $V_{\mathrm{NF}}$ (Fig. 1e, blue curve), as the delay time $\tau$ is

74 varied (red curves labelled 1, 2, 3).

75 The single-molecule switch consists of a single magnesium phthalocyanine $(\mathrm{MgPc})$ molecule 76 adsorbed on a sodium chloride $(\mathrm{NaCl})$ island on a copper substrate. As shown elsewhere ${ }^{40}$, this molecule 77 can be switched back and forth between two equivalent adsorption geometries by sufficiently strong THz 78 fields (Fig. 2a) that trigger tunnelling into the lowest unoccupied molecular orbital (LUMO). We monitor 79 a small non-resonant co-tunnelling current, which is driven by a small DC bias of $\sim 150 \mathrm{mV}$ (see 80 Methods), to decide for every individual laser pulse whether or not the molecule has switched. This way 81 we record the switching rate, $p$, with increasing waveform amplitude (Fig. 2b). When the THz peak 82 voltage $V_{\text {peak }}$ suffices to charge the LUMO, $p$ rapidly rises (Fig. 2c, black dots). This onset behaviour 83 follows an error function (black curve), the derivative of which (grey Gaussian curve) mimics the 84 phonon-broadened (see Methods) LUMO resonance line at $V_{\text {LUMO }}=1.2 \mathrm{~V}$. As this derivative $\mathrm{d} p / \mathrm{d} V_{\text {peak }}$ is 85 the ultrafast analogue of the differential conductance $\mathrm{d} I / \mathrm{d} V$ in steady-state STM, we superimpose the 86 resonance curves obtained by steady-state (blue) and femtosecond spectroscopy (grey) to gauge the tip87 confined $\mathrm{THz}$ peak voltage $V_{\text {peak }}$ in units of volts (vertical axis of Fig. 2c) without any free parameter. 88 Consequently, when we couple the similarly shaped superposition waveforms $V_{\text {sum, } \tau}$ to the STM and 89 detect the THz-induced switching rate, $p$, we retrieve the local peak voltage across the tunnelling junction 90 by utilizing the inverted onset curve $V_{\text {peak }}(p)$. In particular, we note that the switching motion is triggered 91 by a quasi-instantaneous electron tunnel event temporarily confined to the maximum of the gate pulse.

92 The amplitude of the gate pulse was chosen such that the superposition waveforms $V_{\text {sum }, \tau}$ covered the 93 steepest region of $V_{\text {peak }}(p)$, to maximize the sensitivity. By monitoring the switching rate $p(\tau)$ of the 94 molecular switch, we extracted the peak voltages of the superposition waveforms, $V_{\text {peak }}(\tau)$, and thus 95 obtained the first time-resolved atomic-scale femtosecond waveform $V_{\mathrm{NF}}(t)$ (Fig. 3). For the incident far96 field transient detected electro-optically (Fig. 3a) and centred at a frequency of $0.9 \mathrm{THz}$ (Fig. 3a, inset), 97 we retrieve the tip-confined voltage waveform shown in Fig. 3b. These waveforms differ quite markedly. 
98 The tip-confined pulse is longer and exhibits a different carrier-envelope-phase (CEP). Compared to the

99 far-field, the near-field spectrum peaks at a lower frequency around $0.5 \mathrm{THz}$ and exhibits a subtle

100 oscillatory structure (Fig. 3c). The near-field spectral phase (Fig. 3d) is almost as flat as that of the far-

101 field waveform, but the tip-confined pulse exhibits a CEP shift of approximately $-\pi / 3$. The accumulated

102 amplitude and phase effects upon coupling and propagation of a far-field waveform into the tip-confined

103 atomic near field are summarized in a frequency-dependent complex-valued transfer function (Fig. 3e).

\section{Plasmonic coupling of the far field to the tunnelling junction}

105 To scrutinize salient characteristics of this transfer function that arise from classical electrodynamics,

106 we simulated the field propagation by numerically solving Maxwell's equations with a finite-element

107 calculation (see Methods for details). We apply the calculated transfer function (Fig. 3e, solid curves) to

108 the far-field spectrum and obtain a simulation of the tip-confined voltage waveform via an inverse Fourier

109 transform (Fig. 3b, black curve). The shape of the amplitude spectrum is reproduced very well (Fig. 3c).

110 Even the frequency-periodic structure, which stems from a resonator for surface plasmon waves formed

111 by the etched tip geometry (see Methods), is matched. The experimentally observed redshift of the near-

112 field spectrum is caused by the $1 / f$-scaling of the field enhancement. Also, the simulated phase agrees

113 with the measurement remarkably well, including both the curvature of the phase function and the

114 prominent CEP shift of approximately $-\pi / 3$ (Fig. $3 \mathrm{~d}$ ). Comparing the measured and simulated time-

115 domain waveforms (Fig. 3b), the overall shape and even subtle features like the small kink after the main

116 peak match, opening the door to predictive design of coherent control in near-field nanooptics.

117 The transient bias voltage in the simulations is calculated as the product of the field $E_{\mathrm{NF}}$ in the middle

118 of the tunnelling junction times the tunnelling distance $d$. In the regime in which the field enhancement is

119 inversely proportional to the gap size $E_{\mathrm{NF}} \propto 1 / d$, as predicted by the classical antenna theory (see

120 Methods), the voltage drop between tip and substrate $V_{\mathrm{ts}}=E_{\mathrm{NF}} d$ does not depend on the tip height

121 providing the absolute scale to the tip-confined voltage waveform $V_{\mathrm{NF}}(t)$. With this gauge, the classical

122 simulation predicts the correct tip enhancement of as much as $2 \times 10^{5}$. Voltage waveforms acquired at

123 different tip heights (Extended Data Fig. 1a) support this picture and illustrate the reproducibility of our 
124 near field detection. We also note that the shape of the near-field transients we record is robust against 125 mechanical modification of the atomistic tip apex (Extended Data Fig. 1b).

\section{Local femtosecond quantum dynamics}

127 Nonetheless, our experimental approach relies on two implicit assumptions that are questioned if 128 electron tunnelling takes place: (i) The voltage gauge from steady-state spectroscopy requires ultrafast 129 lightwave biasing to drive tunnelling at the same local field strength required for steady-state tunnelling.

130 (ii) The tunnelling-based measurement process should exhibit minimal back-action on the instantaneous

131 voltage, which is to be measured. Recent theoretical simulations of light-matter interaction at atomic 132 scales have shown that light-driven tunnelling currents can significantly modify near fields and lead to 133 retardation effects, owing to accumulating charge imbalance ${ }^{39}$. In this light, the good agreement of our 134 experimental results with classical-electrodynamics simulations that do not even take the presence of the 135 molecule in the junction into account calls for a thorough analysis of the role of generated ultrafast 136 tunnelling currents. Hence, we developed a full quantum mechanical description of the ultrafast 137 dynamical scenario with time-dependent density functional theory (TDDFT). The tip is modelled as a 138 tetrahedral cluster of metal atoms and the molecule is placed at distances of 6 and $9 \AA$ from the tip and the 139 substrate, respectively (Fig. 4a). Time domain simulations at a centre frequency of $0.9 \mathrm{THz}$ used in the 140 experiment are computationally prohibitively expensive. However, we verified (see Supplementary 141 Information for details) that the relevant physical effects can be captured by simulating a much faster 142 waveform oscillating at $40 \mathrm{THz}$ (and $20 \mathrm{THz}$ ), for which the Keldysh parameter remains well below one 143 and the photon energy well below any electronic excitation in the system. We computed the transient 144 current and static and dynamical molecular screening for different field strengths reaching the onset of 145 electron tunnelling into the molecule (around $0.2 \mathrm{~V} / \AA$ ). In what follows, all local observables were plane 146 averaged (see Methods for details).

147 The self-consistent local screening in the molecule as well as electron tunnelling between tip and 148 molecule is shown to significantly alter the local potential and, hence, the near field in the junction, as 149 shown in Figs. 4a,b. The calculated Hartree potential difference with and without the molecule in the 
junction vary locally very strongly (Supplementary Video 1). Following the longitudinal near-field

151 strength averaged in the molecular region over time (Fig. 4c), we find strong retardation effects: The first

152 section of the transient is barely modified but drives tunnelling from the tip into the molecule. The charge

153 accumulation in the molecule together with the hole left behind in the tip leads to an electric field

154 opposite to the one of the largest half-cycle, strongly modifying the remaining near-field transient.

155 In our experiment, the voltage transient is probed by the tunnelling of electrons, which drive the 156 molecule to repeatedly switch its orientation. Thus, it is important for the interpretation of the 157 experimental data, whether the tunnelling currents are driven at similar voltage thresholds as in steady158 state spectroscopy with quasi-static biasing. Remarkably, our first principles TDDFT simulations predict 159 an almost unchanged onset behaviour for the tunnelling currents as a function of the voltage drop across 160 the junction (Fig. 4d), which is exactly what our sensor probes. In addition, retardation effects are not 161 strong enough to drive an appreciable current in the opposite direction and, therefore, do not alter the 162 current-to-peak-voltage relation, which our experiments rely on. Thus, we conclude that, while the near 163 field in the junction is distributed very inhomogeneously, the total peak voltage across the tunnelling 164 junction is probed very accurately by our atomic-scale sensor.

165 Finally, in our sampling scheme the test waveform only adds a small offset to this peak field, which 166 is sensed via single-electron tunnelling. Exploiting this quantum process to gauge the near field is robust:

167 First, the quasi-instantaneous tunnel process is not affected by the subsequent fields or the way they are 168 perturbed by local dynamics. Second, our experiment benefits from the quantum mechanical principle 169 that a particle does not alter its own potential. When one isolated electron tunnels through a potential 170 barrier (Fig. 4e), it leaves its energy landscape unchanged. Nevertheless, an external observer or other 171 particles would sense the electric field of the tunnelling electron and observe a potential being modified 172 by the tunnelling electron. This fundamental contemplation leads us to the conclusion that different 173 regimes have to be discriminated in such experiments: In a regime where less than one electron tunnels on 174 average per laser pulse, we sense a voltage transient that is unperturbed by the tunnelling process itself. 175 This is the limit in which the present experiments have been conducted. On the other hand, if many 
176 electrons tunnel during every single pulse transient, the tunnelling electrons may act back on all other

177 electrons, giving rise to a vastly modified potential. In this scenario, the behaviour may not be quasi-

178 static. In an intermediate regime of few tunnelling electrons, even pulse-to-pulse quantum fluctuations

179 may have to be taken into account.

180 Outlook

181 Our novel approach opens several new doors across nanoscience and nanophotonics at once. Most

182 fundamentally, with this local sampling scheme, atomic-scale near fields can now be spatio-temporally

183 mapped. We expect precisely calibrated movies of sub- $\AA$ field distributions to reveal the limits of

184 classical nanooptics and directly visualize the quantum nature of atomic-scale light-matter interaction.

185 Moreover, state-of-the-art simulations that bridge the gap between macroscopic light and atomic

186 waveforms can now be gauged by experiment, and thereby revolutionize the design of nanotechnology

187 such that novel metamaterials and atomic-scale devices make use of precisely tailored coherent near

188 fields, faster than a cycle of light.

$189{ }^{\#}$ These authors contributed equally to this work.

190 *Authors to whom correspondence should be addressed. 
191 Author Contributions D.P., C.R., L.Z.K., T.B., A.N., J.H., J.R. and R.H. conceived, set up and carried 192 out the experiments. D.P. and A.N. implemented and carried out the classical finite-element simulations. 193 F.B., D.S., M.R., and A.R. conceived, implemented and carried out the TDDFT simulations. All authors 194 analysed the data and wrote the manuscript.

195 Data Availability The data that support the plots within this paper and other findings of this study are 196 available from the corresponding authors upon reasonable request.

197 Additional Information Reprints and permissions information is available at www.nature.com/reprints.

198 The authors declare no competing financial interests. Correspondence and requests for materials should 199 be addressed to A.R. (angel.rubio@mpsd.mpg.de) or R.H. (rupert.huber@physik.uni-regensburg.de). 


\section{References}

201 1. Wu, Q. \& Zang, X.-C. Free-space electro-optic sampling of terahertz beams. Appl. Phys. Lett. 67, $202 \quad 3523-3525$ (1995).

203 2. Kübler, C., Huber, R., Tübel,S. \& Leitenstorfer, A. Ultrabroadband detection of multi-terahertz

204 field transients with GaSe electro-optic sensors: Approaching the near infrared.

205 Appl. Phys. Lett. 85, 3360-3362 (2004).

206 3. Liu, W. et al. A fully reconfigurable photonic integrated signal processor. Nat. Photon. 10, 207 190-195(2016).

208 4. Sederberg, S. et al. Attosecond optoelectronic field measurement in solids. Nat. Commun. 11, 430 $209 \quad$ (2020).

210 5. Paul, P. M. et al. Observation of a Train of Attosecond Pulses from High Harmonic Generation. $211 \quad$ Science 292, 1689-1692 (2001).

212 6. Huber, R. et al. How many-particle interactions develop after ultrafast excitation of an electron213 hole plasma. Nature 414, 286-289 (2001).

214 7. Drescher, M. et al. Time-resolved atomic inner-shell spectroscopy. Nature 419, 803-807 (2002).

215 8. Kienberger, R., Goulielmakis, E., Uiberacker, M. et al. Atomic transient recorder. Nature 427, $216 \quad 817-821(2004)$.

217 9. Dudovich, N. et al. Measuring and controlling the birth of attosecond XUV pulses.

$218 \quad$ Nat. Phys. 2, 781-786 (2006).

219 10. Corkum, P. B. \& Krausz, F. Attosecond science. Nat. Phys. 3, 381-387 (2007).

220 11. Goulielmakis, E. et al. Attosecond Control and Measurement: Lightwave Electronics. Science 317, $221 \quad 769-775(2007)$.

222 12. Eisele, M. et al. Ultrafast multi-terahertz nano-spectroscopy with sub-cycle temporal resolution. $223 \quad$ Nat. Photon. 8, 841-845 (2014).

224 13. Basov, D. N., Fogler, M. M. \& García De Abajo, F. J. Polaritons in van der Waals materials. $225 \quad$ Science 354, aag1992 (2016). 
226 14. Merlein, J. et al. Nanomechanical control of an optical antenna.

227 Nat. Photon. 2, 230-233 (2008).

228 15. Liu, M. et al. Terahertz-field-induced insulator-to-metal transition in vanadium dioxide

229 metamaterial. Nature 487, 345-348 (2012).

230 16. Yoshida, K., Shibata, K. \& Hirakawa, K. Terahertz field enhancement and photon-assisted

231 tunneling in single-molecule transistors. Phys. Rev. Lett. 115, 138302 (2015).

232 17. Spektor, G. et al. Revealing the subfemtosecond dynamics of orbital angular momentum in

233 nanoplasmonic vortices. Science 355, 1187-1191 (2017).

234 18. Esmann, M. et al. Vectorial near-field coupling. Nat. Nanotechnol. 14, 698-704 (2019).

235 19. Seo, M. A. et al. Terahertz field enhancement by a metallic nano slit operating beyond the skin236 depth limit. Nat. Photon. 3, 152-156 (2009).

237 20. Savage, K. J. et al. Revealing the quantum regime in tunnelling plasmonics.

$238 \quad$ Nature 491, 574-577 (2012).

239 21. Benz, F. et al. Single-molecule optomechanics in 'picocavities'. Science 354, 726-729 (2016).

240 22. Krüger, M., Schenk, M., Hommelhoff, P. \& Kruger, M. Attosecond control of electrons emitted 241 from a nanoscale metal tip. Nature 475, 78-81 (2011).

242 23. Herink, G., Solli, D. R., Gulde, M. \& Ropers, C. Field-driven photoemission from nanostructures 243 quenches the quiver motion. Nature 483, 190-193 (2012).

244 24. Förg, B. et al. Attosecond nanoscale near-field sampling. Nat. Commun. 7, 11717 (2016).

245 25. Rybka, T. et al. Sub-cycle optical phase control of nanotunnelling in the single-electron regime.

246 Nat. Photon. 10, 667-670 (2016).

247 26. Barnes, W. L., Dereux, A. \& Ebbesen, T. W. Surface plasmon subwavelength optics.

$248 \quad$ Nature 424, 824-830 (2003).

249 27. Cocker, T. L. et al. An ultrafast terahertz scanning tunnelling microscope.

$250 \quad$ Nat. Photon. 7, 620-625 (2013). 
251 28. Cocker, T. L., Peller, D., Yu, P., Repp, J. \& Huber, R. Tracking the ultrafast motion of a single 252 molecule by femtosecond orbital imaging. Nature 539, 263-267 (2016).

253 29. Yoshioka, K. et al. Real-space coherent manipulation of electrons in a single tunnel junction by 254 single-cycle terahertz electric fields. Nat. Photon. 10, 762-765 (2016).

255 30. Jelic, V. et al. Ultrafast terahertz control of extreme tunnel currents through single atoms on a 256 silicon surface. Nat. Phys. 13, 591-597 (2017).

257 31. Yoshioka, K. et al. Tailoring Single-Cycle Near Field in a Tunnel Junction with Carrier-Envelope 258 Phase-Controlled Terahertz Electric Fields. Nano Lett. 18, 5198-5204 (2018).

259 32. Li, S., Chen, S., Li, J., Wu, R. \& Ho, W. Joint Space-Time Coherent Vibration Driven 260 Conformational Transitions in a Single Molecule. Phys. Rev. Lett. 119, 176002 (2017).

261 33. Böckmann, H. et al. Near-Field Enhanced Photochemistry of Single Molecules in a Scanning 262 Tunneling Microscope Junction. Nano Lett. 18, 152-157 (2018).

263 34. Lee, J., Crampton, K. T., Tallarida, N. \& Apkarian, V. A. Visualizing vibrational normal modes of 264 a single molecule with atomically confined light. Nature 568, 78-82 (2019).

265 35. Kubo, A., Pontius, N. \& Petek, H. Femtosecond Microscopy of Surface Plasmon Polariton Wave 266 Packet Evolution at the Silver/Vacuum Interface. Nano Lett. 7, 470-475 (2007).

267 36. Barbry, M. et al. Atomistic Near-Field Nanoplasmonics: Reaching Atomic-Scale Resolution in 268 Nanooptics. Nano Lett. 15, 3410-3419 (2015).

269 37. Rácz, P. et al. Measurement of Nanoplasmonic Field Enhancement with Ultrafast Photoemission. $270 \quad$ Nano Lett. 17, 1181-1186 (2017).

271 38. Yoshida, S. et al. Subcycle Transient Scanning Tunneling Spectroscopy with Visualization of 272 Enhanced Terahertz Near Field. ACS Photonics 6, 1356-1364 (2019).

273 39. Jestädt, R., Ruggenthaler, M., Oliveira, M. J. T., Rubio, A. \& Appel, H. Light-matter interactions 274 within the Ehrenfest-Maxwell-Pauli-Kohn-Sham framework: fundamentals, implementation, and 275 nano-optical application. Adv. Phys. 68, 225-333 (2019). 
276 40. Peller, D. et al. Sub-cycle atomic-scale forces coherently control a single-molecule switch. $277 \quad$ Nature 585, 58-62 (2020). 
278 Acknowledgements We thank Christian Meineke, Andreas Pöllmann, Christoph Rohrer, and Martin 279 Furthmeier for assistance and Ferdinand Evers, Heiko Appel and Sebastian Ohlman for discussions. 280 Financial support from the Deutsche Forschungsgemeinschaft (DFG, German Research Foundation) 281 through Project-ID 314695032 - SFB 1277 (Subproject B02), Research Grants HU1598/3 and HU1598/8, 282 the Cluster of Excellence "Advanced Imaging of Matter" (AIM, EXC 2056, ID 390715994) and from 283 Grupos Consolidados (IT1249-19), the European Research Council (ERC-2015-AdG694097), the 284 European Union's Horizon 2020 research and innovation programme under the Marie Skłodowska-Curie 285 grant agreement No. 895747, and the Flatiron Institute, a division of the Simons Foundation is gratefully 286 acknowledged. 
288 STM setup. The homebuilt $\mathrm{STM}^{28}$ operates at ultrahigh vacuum (UHV; pressure $\sim 7 \times 10^{-11}$ mbar) and 289 cryogenic $(7 \mathrm{~K})$ conditions. The bias voltage is applied to the sample. A homebuilt high-gain $290\left(\mathrm{G}=2.5 \times 10^{10} \mathrm{~V} / \mathrm{A}\right)$ preamplifier $(I-V$-converter $)$ is mounted close to the STM head. The collimated THz 291 beam enters the vacuum chamber through a sapphire viewport and is focused onto the tunnel junction by 292 a parabolic mirror that is mounted on the STM head.

293 THz optical setup. Phase-locked THz pulses (centre frequency, $0.9 \mathrm{THz}$ ) are generated by tilted-pulse294 front optical rectification of femtosecond near-infrared pulses (1028 nm centre wavelength, $250 \mathrm{fs}$ pulse 295 duration FWHM) from a regenerative laser amplifier (repetition rate tuneable from $0.61 \mathrm{MHz}$ down to 296 single shot) in lithium niobate. Pairs of mutually delayed THz transients are prepared by transmitting the $297 \mathrm{THz}$ pulses through a Michelson interferometer, in which the computer-controlled position of one end 298 mirror sets the delay time, $\tau$. Crossed wire grid polarizers allow us to continuously tune the THz field 299 amplitude, for both pulses individually, without changing the waveform. To retrieve the far-field 300 waveforms that are incident in the STM junction, we mimic the aperture of the vacuum chamber on the 301 optical table.

302 Single-molecule switch. Sodium chloride $(\mathrm{NaCl})$ is evaporated thermally onto a clean $\mathrm{Cu}(111)$ surface 303 under UHV conditions at $275 \mathrm{~K}$. Subsequently, at a sample temperature below $15 \mathrm{~K}$, magnesium 304 phthalocyanine molecules are deposited to adsorb on chlorine sites of a $\mathrm{NaCl}$ surface such that the 305 molecular and substrate symmetry directions are rotated with respect to each other, resulting in two 306 degenerate, stable adsorption geometries ${ }^{41}$. As studied elsewhere, transient charging of the molecule by 307 lightwave-induced injection of a single electron into the LUMO induces switching events between the 308 two adsorption geometries with a certain probability ${ }^{40}$. The onset of LUMO tunnelling, which depends on 309 the material composition of the substrate ${ }^{41}$, has been shown to remain the same for terahertz-induced and 310 DC electron tunnelling ${ }^{40}$. Here, the differential conductance associated to LUMO tunnelling is peaked 311 around a bias voltage of $1.2 \mathrm{~V}$ and strongly Gaussian-broadened owing to Coulomb coupling between the 312 temporary excess charge in the molecule and the ions in the $\mathrm{NaCl}$ thin film - a phenomenon well known 
313 from steady-state STM experiments ${ }^{42}$. Furthermore, it has been demonstrated that the lightwave-driven 314 switching probability is proportional to the rate of lightwave-triggered electron tunnelling into the $315 \mathrm{LUMO}^{40}$. At specific tip positions, the ultrafast atomic force associated with the near field can also be 316 used to trigger a coherent librational motion of the molecule, which can coherently modulate the 317 switching rate $^{40}$. By performing the experiments at a selected spatial location over the molecule, we 318 managed to minimize this influence ${ }^{40}$, which is undesirable in the present context, to below the 319 uncertainty margin. Varying the $\mathrm{THz}$ field strength allows us to spectroscopically access the phonon320 broadened LUMO resonance and detect peak-field-dependent switching rates as shown in Fig. 2c and 321 when sampling near-field waveforms.

322 During each THz transient, the electric field remains far below the bias threshold for resonant tunnelling, 323 except for a time window of $\sim 100$ fs during the field crest of the most intense half-cycle of the gate 324 transient. After this time window has passed, no further resonant tunnelling occurs that could possibly be 325 influenced by the previous event. Hence, this restriction to a maximum of one single tunnelling event per 326 THz transient ensures minimal back-action.

327 We monitor every switching event by a non-resonant detection current to directly retrieve the switching 328 probability. The detection current is induced by a small bias voltage on the order of $150 \mathrm{mV}$, far below 329 the voltage required for LUMO tunnelling and therefore too small to charge the molecule ${ }^{40}$. Hence this 330 current stems from electrons tunnelling directly between tip and substrate, a process also denoted "non331 resonant cotunnelling".

332 We note that the presented sampling scheme can in principle be employed in many other scenarios where 333 an observable depends monotonically on the tip-confined field. Instead of the MgPc molecular switch, 334 which we utilized as a near-field detector due to its very strong response, for example a tunnelling current 335 into any conductive sample exhibiting a spectrally sharp nonlinear response, or photoluminescence 336 detected externally could be considered as alternative sensing strategies. For the detection of particularly 337 low field strengths even a superconducting sample could be conceivable as a detector. 
338 Statistical uncertainty in the detection of the switching probability. In order to resolve the switching

339 probability - and therefore the near-field waveform - with high precision, the experiments are repeated

340 numerous times to observe a sufficient number of events for every set of parameters (field strength for the

341 calibration curve, delay time for the near-field waveform). In Fig. 3b, for example, every data point

342 represents statistics extracted from an observation period where a total of around 1 million pulse pairs

343 were coupled to the STM. This sequence triggered about $n p=1000$ switching events. Statistically, every

344 laser shot represents a Bernoulli trial and every sequence follows a binomial distribution. This yields a

345 shot-noise limited signal-to-noise (SNR) ratio of $\sqrt{n p}=33$ corresponding to a relative statistical

346 uncertainty of $3 \%$, which is the dominating source of experimental uncertainty. By increasing the number

347 of observed events the uncertainty margin could be reduced even further.

348 Classical finite-element simulations of the sampled near-field THz waveforms. We performed

349 numerical simulations using the frequency-domain finite element solver COMSOL. Maxwell's equations

350 are solved for complex-valued electromagnetic fields on a discrete mesh, providing a self-consistent

351 three-dimensional map of amplitude and phase distributions. All classical wave propagation, plasmonic

352 coupling to the tip shaft, screening and localization in the $\AA$-sized tip-sample gap are accounted for via

353 the complex-valued dielectric properties of the materials used in the experiment.

354 The geometry is modelled within a cuboid cell where the incident electromagnetic radiation is

355 implemented as oscillatory boundary conditions on one side of the simulation volume. The shape of the

356 tungsten tip is chosen based on actual electron microscope images (Extended Data Fig. 2a,b). It consists

357 of a cylindrical wire with a diameter of $200 \mu \mathrm{m}$ and a conical, etched region with a $15^{\circ}$ taper and a height

358 of $200 \mu \mathrm{m}$. The apex features a radius of curvature of $300 \mathrm{~nm}$. A flat gold sample is placed at a distance

359 of $1 \mathrm{~nm}$ below the tip. The dielectric functions used for tungsten and gold are adopted from the

360 literature ${ }^{43,44}$. The physical simulation volume is surrounded by perfectly matched layers (PML) as

361 boundaries on 4 sides imitating an infinite world by suppressing all back-reflections of radiation. The

362 beam enters the simulation volume through one cell wall at an angle of $40^{\circ}$ relative to the sample surface. 
363 The excitation boundary absorbs plane waves leaving the simulation volume by fulfilling the scattering 364 boundary condition.

365 In order to resolve the propagation of electromagnetic waves with millimetre wavelength from the far 366 field down to Å-scale localized near fields, the simulation cell is partitioned into a graded mesh consisting 367 of tetrahedrals with sizes between $25 \mu \mathrm{m}$ and $5 \AA$. At all metal surfaces, the mesh is chosen fine enough 368 to resolve skin depth effects. The boundary PMLs are meshed with hexahedra and the tip and sample 369 extend vertically through them. The junction was meshed with at least two grid points in between tip and 370 sample surface. A direct matrix solver computes the solution to a predefined tolerance level for each 371 frequency. To cover the entire spectrum of the transient, the simulated frequencies range from $0.33 \mathrm{THz}$ 372 to $3.33 \mathrm{THz}$ with a linear spacing of $33 \mathrm{GHz}$.

373 Extended Data Figure 2c shows the simulated field distribution about the tip upon plane-wave excitation.

374 In the top left corner of the panel, the incident plane waves propagate towards the junction with constant 375 amplitude, hardly influenced by the geometry. Closer towards the substrate, the wave fronts appear 376 transversally structured, which stems from an interference with the reflection off the flat metal substrate.

377 In proximity to the tip apex, pronounced field enhancement and phase retardation effects manifest. 378 Directly in the tunnelling gap, we find a field enhancement factor of $\sim 2 \times 10^{5}$ for a tip-sample distance of $3791 \mathrm{~nm}$ (Extended Data Fig. 2d). According to classical antenna theory, the field enhancement is inversely 380 proportional to the gap size $E_{\mathrm{NF}} \propto 1 / d($ ref. 45$)$.

381 To remove artefactual diffraction from the simulation, we performed a second calculation with the same 382 simulation volume and mesh, but we replaced the entire geometry with bare vacuum. Comparing the 383 complex electric field of both scenarios allowed us to obtain the complex transfer function shown in 384 Extended Data Figure 2e. The field-enhancement amplitude (black solid line) approximately scales with 385 the inverse frequency, $f^{-1}$. Both amplitude and phase of the simulated transfer function exhibit a minor 386 oscillatory structure with a periodicity of $\sim 1.3 \mathrm{THz}$, the origin of which is revealed by the simulated 387 pattern of spatial field distribution (Extended Data Fig. 3). The curved part of the tip that converges 388 towards the apex has been manufactured by electrochemical etching. For the employed tip shown in 
389 Extended Data Figure 2a,b, this process created a sharply curved edge at the circumference where etching 390 began. As this edge serves as a reflector for surface plasmons, a standing-wave pattern can form vertically 391 across the $\sim 250 \mu \mathrm{m}$ etched region, giving rise to a periodic structure of frequencies with slightly 392 amplified or attenuated coupling efficiency (Extended Data Fig. 3b-d).

393 Ab initio TDDFT simulations. We performed the real-time TDDFT calculations with the Octopus $394 \operatorname{code}^{46,47}$ employing the adiabatic local density approximation (LDA) ${ }^{48}$ to describe exchange-correlation 395 effects. In order to obtain an efficient and accurate description of the relatively long-range interactions 396 between substrate, tip and molecule, the averaged density self-interaction correction was applied ${ }^{49}$. To 397 ensure the stability of our time-propagation, we solved the time-dependent Kohn-Sham equations self398 consistently at every time step using the enforced time-reversal symmetry propagator ${ }^{50}$. We employ 399 norm-conserving pseudo-potentials to describe the core-valence interactions. We constructed a 400 microscopic model system consisting of a sodium substrate-tip geometry with and without the confined $401 \mathrm{MgPc}$ molecule according to Fig. 4a and b, respectively. The substrate was modelled as a finite slab of 402256 atoms, and a tetrahedral structure of 55 atoms was used to represent the STM tip, located on top of a 403 phenyl $\mathrm{H}$ atom of the $\mathrm{MgPc}$ molecule. The use of $\mathrm{Na}$ instead of $\mathrm{Cu}$ and $\mathrm{W}$ considerably reduces the 404 computational cost, but we confirmed that it reproduces the proper physical description of a metallic STM 405 junction under a non-resonant, THz-range perturbation, giving access to the longer time-scale dynamics. 406 The MgPc geometry was optimized on a $\mathrm{NaCl}$ island to account for the presence of the distance spacer ${ }^{40}$. 407 In the dynamical simulations the $\mathrm{NaCl}$ layers were replaced by vacuum space of the same height ( $9 \AA)$. 408 Local observables (electric fields, charge and current) were averaged over the whole $x-y$ plane and in a $409 \quad 3.7-\AA$ region in the z-axis around the centre of the molecule.

410 Numerically, the system was represented on a real space grid with 0.4 atomic units spacing and time411 propagated with using a time-step of 2.15 as until a total time of $55 \mathrm{fs}$ was reached. The external 412 perturbation was represented by a $40 \mathrm{THz}$ waveform (period of $25 \mathrm{fs}$ ), modulated by a Gaussian envelope $413 \quad($ FWHM $=18 \mathrm{fs})$, considering different peak field strengths. The field strength range was such that it 414 spanned values lower and higher than the onset for electron tunnelling into the molecular orbitals, which 
415 is around $0.2 \mathrm{~V} / \AA$. A higher frequency than in the experiment was considered to run simulations in a 416 reasonable computation time at the affordable computational cost (320 cores). However, as vibrational 417 excitations are supressed (ions are clamped in our simulations), and the plasmonic modes have energies ${ }^{39}$ 418 of the order of $1 \mathrm{eV}$, our approximated approach is accurate. The transient current under a (quasi) DC 419 external bias was obtained considering a static external field with a smoothed switch-on ramp of 3 fs 420 duration, modulated by a sine-squared envelope. This microscopic approach to obtain the DC response 421 from real-time dynamics has been proposed and applied previously ${ }^{51}$. The orbital current is calculated by 422 linear regression of the charge dynamics after the end of the initial ramp, subtracting the reference charge 423 dynamics of the junction without molecule. 


\section{Methods References}

425 41. Patera, L. L., Queck, F., Scheuerer, P., Moll, N. \& Repp, J. Accessing a charged intermediate state 426 involved in the excitation of single molecules. Phys. Rev. Lett. 123, 016001 (2019).

427 42. Repp, J., Meyer, G., Paavilainen, S., Olsson, F. \& Persson, M. Phys. Rev. Lett. 95, 225503 (2005).

428 43. Ordal, M. A. et al. Optical properties of the metals $\mathrm{Al}, \mathrm{Co}, \mathrm{Cu}, \mathrm{Au}, \mathrm{Fe}, \mathrm{Pb}, \mathrm{Ni}, \mathrm{Pd}, \mathrm{Pt}, \mathrm{Ag}$, Ti, and $429 \quad \mathrm{~W}$ in the infrared and far infrared. Appl. Optics 22, 1099-1119 (1983).

430 44. Olmon, R. L. et al. Optical dielectric function of gold. Phys. Rev. B 86, 235147 (2012).

431 45. Kang, J. H., Kim, D. S. \& Park, Q.-H. Local Capacitor Model for Plasmonic Electric Field 432 Enhancement. Phys. Rev. Lett. 102, 093906 (2009).

433 46. Tancogne-Dejean, N. et al. Octopus, a computational framework for exploring light-driven 434 phenomena and quantum dynamics in extended and finite systems. J. Chem. Phys. 152, 124119 $435 \quad$ (2020).

436 47. Andrade, X. et al. Real-space grids and the Octopus code as tools for the development of new 437 simulation approaches for electronic systems. Phys. Chem. Chem. Phys. 17, 31371-31396 (2015).

438 48. Perdew, J. P. \& Zunger, A. Self-interaction correction to density-functional approximations for 439 many-electron systems. Phys. Rev. B 23, 5048-5079 (1981).

440 49. Legrand, C., Suraud, E. \& Reinhard, P. G., Comparison of self-interaction-corrections for metal $441 \quad$ cluster, J. Phys. B 35, 1115-1128 (2002).

442 50. Castro, A., Marques, M. A. L. \& Rubio, A. Propagators for the time-dependent Kohn-Sham 443 equations. J. Chem. Phys. 121, 3425-3433 (2004).

444 51. Di Ventra, M. \& Todorov, T. N. Transport in nanoscale systems: the microcanonical versus grand445 canonical picture, J. Phys.: Condes. Matter 16, 8025-8034 (2004). 
446 Figure 1 | Near-field waveform sampling by superposition. When a THz waveform is coupled to a 447 nanotip tunnelling junction (a), its magnitude and shape change drastically in the near field such that the 448 transient voltage $V_{\mathrm{NF}}(t)$ induced at atomistic distances is a priori unknown (b). When a weak test 449 waveform $V_{\mathrm{NF}}(t)(\mathbf{c})$ and a delayed, comparatively strong gate waveform $V_{\text {gate }}(t-\tau)$ (d, labelled 1, 2, 3 for 450 different delay times $\tau$ ) interfere in the near field, the test waveform is imprinted on the sum waveforms $451 V_{\text {sum }}(t)$ (e, red curves labelled as in $\mathbf{d}$ ) as an envelope for the sub-cycle waveform crests (blue curve). 
452 Figure 2 | Quantitative single-molecule peak-voltage sensor. a, A single-molecule switch is used for 453 detection and parameter-free calibration of the near-field peak voltage. When the peak field accesses an 454 orbital resonance, electron tunnelling statistically causes a switching event, which is registered for every 455 laser shot. Due to the phonon-broadened linewidth of the tunnelling resonance, tip-confined voltage 456 waveforms with different peak fields (b) induce different electron tunnelling rates, such that the resulting 457 switching probability $p$ directly encodes the localized peak voltage $V_{\text {peak }}$ (c, data points). This calibration 458 curve describes an error function (black line), the derivative of which ( $\mathrm{d} p / \mathrm{d} V_{\text {peak }}$, grey Gaussian curve) 459 represents the ultrafast analogue of steady-state $\mathrm{d} I / \mathrm{d} V$-spectroscopy (blue curve). Relating the LUMO 460 resonance peaks centred at $1.2 \mathrm{~V}$ allows us to quantify the tip-confined peak voltage directly in units of 461 Volts without any free parameter. For every waveform measurement, a separate calibration curve similar 462 to Fig. 2c was acquired. 
463 Figure 3 | Calibrated atomic-scale near-field waveform. a, A cosine-like far-field waveform with a 464 spectrum centred at $0.9 \mathrm{THz}$ and a flat phase of $\sim 0 \mathrm{rad}$ (inset) is coupled to the nanotip. $\mathbf{b}$, The evolution 465 of the induced near-field voltage is remarkably different (data points). Its overall shape possesses a 466 different CEP and subtle sub-cycle features like a kink at $t=0.6 \mathrm{ps}$ are resolved, indicating a more 467 structured spectrum. c, Indeed, the spectral amplitude of the measured near-field waveform (data points) 468 is shifted to lower frequencies and possesses a minor oscillatory structure with a period of $1.3 \mathrm{THz}$. d, A 469 similar slight modulation is visible in the spectral phase (data points), which is otherwise flat at 470 approximately -1 rad. e, Relating the near-field and far-field spectra and assuming a locally homogeneous 471 electric field across a tip-sample distance of $10 \AA$ yields the complex transfer function that visualizes the $472 f^{-1}$-like field enhancement and CEP shift of approximately $-\pi / 3 \mathrm{rad}$. A classical electrodynamic 473 simulation qualitatively reproduces these results including fine details, without any free parameter (black 474 lines in b-e, see Methods for details). 
475 Figure 4 | Quantum-mechanical simulation of atomic-scale light-matter interaction. In the time-

476 dependent DFT calculations, the junction is modelled as a tetrahedral tip above four atomic substrate

477 layers with and without the phthalocyanine molecule. Lightwaves are modelled as an ultrafast z-polarized

478 external field transient (see Methods for details). a,b, A vertical cross section of the dynamical Hartree

479 potential at the field maximum (relative to the potential at the apex atom at $t=-25 \mathrm{fs}$ ) reveals

480 inhomogeneous near fields, strongly localized around the front-most apex atom. Including the molecule

481 (a) alters the near-field distribution at angstrom scales. c, The time-dependent vertical electric field

482 component, $E_{\mathrm{z}}$, averaged across the molecular plane, is, thus, also modified by the presence of the

483 molecule by roughly a factor of 2 . Accumulation of transferred charge leads to retardation effects and a

484 trailing field offset. d, Despite this back-action on the local fields, the onset behaviour of lightwave-

485 driven tunnelling and of steady-state tunnelling are similar, confirming that our experimental calibration

486 of the local transient voltage remains valid. e, A schematic picture of tunnelling (symbolic electron

487 wavefunction in blue) illustrates that deeply in the single-electron tunnelling regime, the electron wave

488 packet does not affect its own potential landscape (black), warranting minimal back-action of our field-

489 measurements on the near field. 